\title{
M odelo sistémico de mejora continua para la optimización de procesos académicos en universidades privadas
}

\author{
A cuña Salinas, Erika Inés ${ }^{1}$ \\ ${ }^{1}$ Universidad Peruana Unión (U PeU), Perú
}

Recibido el 20 de junio de 2014 - A ceptado el 28 de octubre de 2014

\begin{abstract}
Resumen
A lo largo de las dos últimas décadas en el escenario de la educación superior latinoamericana han surgido nuevas demandas educativas, nuevos modelos institucionales diversos y flexibles, la necesidad de fortalecimientos de políticas de ingreso estudiantil, formación didáctica de los profesores, la articulación con otros niveles educativos, renovación y fortalecimiento de las formas de gobierno universitario, aseguramiento de la calidad académica. Desde este panorama, las universidades privadas adventistas de la red sudamericana vienen buscando mejoras en sus formas de operar. En esta línea de investigación se aplicó el modelo sistémico de mejora continua para la optimización de procesos académicos. El plan de mejora continua se organizó considerando la metodología de sistemas suaves en torno a sus siete etapas aplicadas al sistema académico de la Universidad Peruana U nión, como un sistema prototipo de la red de universidades adventistas del cono sudamericano. En este contexto, el objeto de estudio fue los procesos académicos de la Universidad Peruana Unión. Los objetivos alcanzados se centraron en el estudio de las características funcionales en los aspectos estratégicos, de estructura, de procesos intermedios y de resultado de los procesos académicos de matrícula, de evaluación docente, evaluación discente y de graduación. El propósito de la encuesta de inicio fue realizar el análisis exploratorio para encontrar las fallas, y luego a partir de ese diagnóstico plantear las correcciones. Las muestras de estudiantes y docentes estuvieron conformadas por 307 y 96 sujetos respectivamente, son muestras estratificadas atendiendo a la facultad en la que estuvieron matriculados 0 adscritos. El tipo de investigación corresponde a una investigación aplicada al campo académico tecnológico. El análisis del tratamiento estadístico se basó en el análisis exploratorio de datos (EDA) y para la prueba de hipótesis la prueba T para muestras relacionadas. Las conclusiones sobre la incidencia del modelo sistémico de mejora continua al término de su aplicación se resume: el modelo es eficiente en la optimización de los procesos de matrícula, proceso de evaluación docente, proceso de graduación y el proceso de evaluación discente: caso Universidad Peruana U nión.
\end{abstract}

Palabras dave procesos académicos, optimización, mejora continua, metodología de sistemas blandos.

\begin{abstract}
Over the past two decades on the stage of Latin A merican higher education has created new educational demands, new diverse and flexible business models, the need for fortifications policy of student admission, educational teacher training, coordination with other levels education, renewal and strengthening forms of university governance, academic quality assurance. From this overview, A dventist private universities in the South A merican network come seeking improvements in their ways of operating. In this research the systemic model of continuous improvement to optimize academic processes applied. The improvement plan was organized by considering the soft systems methodology around seven steps applied to the academic system of the Peruvian Union University, as a prototype of the network of Adventist universities in the South A merican cone system. In this context, the aim of the study was the academic processes of Peruvian Union U niversity. The objectives achieved focused on the study of the functional characteristics on the strategic aspects of structure, processes and intermediate outcomes of academic enrollment processes, teacher evaluation, assessment, learning and graduation. The purpose of the survey was to conduct initial exploratory analysis to find flaws, and then from that pose diagnostic corrections. Samples of students and teachers were made up of 307 and 96 subjects, respectively, stratified samples are attending faculty who were enrolled in or assigned. The research is for applied research to technological academic field. The analysis of the statistical treatment was based on exploratory data analysis (EDA ) and the hypothesis test T test for related samples. The findings on the incidence of systemic model of the term continuous improvement of its implementation is summarized: the model is efficient in optimizing processes tuition teacher evaluation process, ranking process and the evaluation process, learning: case Universidad Peruana Union.
\end{abstract}

Keywords: academic, optimization, continuous improvement, soft systems methodology .

\section{Introducción}

Investigar sobre la optimización de los procesos académicos de una institución educativa superior, considerando un plan de mejoramiento continuo, tiene gran complejidad por la diversidad de modelos y perspectivas teóricas desde que se analiza esta problemática, la presencia de un conjunto amplio de

Correspondencia al autor: 
factores y actores que participan en el proceso, la rapidez de la innovación de las tecnologías de comunicación, el aumento de la competitividad y de mayores exigencias de los estudiantes y docentes, entre otros. Esta preocupación encaja en una visión más holística: la innovación como motor de diferenciación. Que las universidades se diferencien más entre ellas gracias a la innovación, que tengan más capacidad de captar talento, estudiantes, recursos y procesos desde un modelo innovador diferenciador. Que el reto de la educación superior sea construir nuevas oportunidades en el ámbito académico; nuevos formatos de estudios, nuevos modelos de alianzas, nuevos modelos de aprendizaje, en el área de la investigación (open science) 0 en el campo de la transferencia de conocimiento.

Entre los trabajos más relevantes se destacan los de Coelho, Romero y Yáber (2005), quienes proponen diseñar, implantar y evaluar indicadores de gestión en el Programa de Especialización en Gerencia de la Empresa, empleando una visión sistémica de la organización y la gerencia de sistemas conductuales como enfoques para desarrollar indicadores de desempeño clave para programas académicos de posgrado; Garzon (2012) reporta la aplicación del proyecto de calidad y mejora continua-PQiMC dirigido a apoyar la gestión de mejora continua para lograr la excelencia de los centros de formación profesional reglada, en el curso 2009-2010 de Cataluña, España. También tenemos la contribución de Suárez (2008) cuyo estudio se enmarcó y se orientó en el estudio de dos aspectos: aumentar la comprensión de la sostenibilidad de la mejora continua de procesos en las administraciones públicas, específicamente a nivel de los ayuntamientos de España, y formular marcos 0 esquemas conceptuales teóricos de la sostenibilidad de la mejora continua de procesos en las administraciones locales.

Los siguientes criterios adoptados justifican la realización de la investigación: valorar el uso de la metodología de sistemas blandos en la mejora continua de los procesos académicos de la Universidad Peruana Unión como prototipo, en el sentido de que es la universidad mayor de la red de universidades adventistas del cono sur de Sudamérica; garantizar la permanencia de la funcionalidad de los procesos académicos en el quinquenio 2012 - 2017, potenciar la producción y gestión de nuevos conocimientos a través de la optimización de los procesos académicos, detección de situaciones problemáticas en procesos académicos que están pasando desapercibidos y que a lo largo y mediano plazo puedan generar problemas más grandes.

La hipótesis principal de esta investigación es probar que el modelo sistémico de mejora continua es eficiente en la optimización de los procesos académicos en universidades privadas de la red adventista sudamericana: caso U niversidad Peruana U nión.

Esta investigación está constituida por cinco capítulos estructurados de la siguiente manera: Se dedica el primer capítulo de este trabajo al planteamiento del problema de investigación, los antecedentes, los objetivos, la justificación, limitaciones, entre otros aspectos.

En el segundo capítulo se aborda los fundamentos del modelo sistémico, el concepto de proceso académico, proceso de transformación, proceso de mejora continua, el ciclo PHVA, marco conceptual y formulación de las hipótesis. En el tercer capítulo se observa la parte operativa de la aplicación del modelo sistémico de mejora continua para la optimización de los procesos académicos: caso UPeU. En el cuarto capítulo se define el tipo y diseño de investigación, estrategia de la prueba de hipótesis, variables de estudio, muestra, fiabilidad de los instrumentos, y las técnicas de recolección, procesamiento y análisis de datos. En el quinto capítulo se detalla el análisis exploratorio de los resultados y la prueba de hipótesis estadística de la optimización de los procesos académicos. Finalmente en el capítulo seis, abordamos la discusión de los resultados obtenidos, las conclusiones y recomendaciones, terminando con las referencias bibliográficas.

\section{Método}

El presente estudio se enmarca dentro una investigación aplicada en el campo administrativo tecnológico, pues es la utilización de la conjunción de la Metodología de sistemas blandos de Checkland (1992) con el modelo de mejora continua de procesos de Deming (1989) en la optimización de procesos académicos, en provecho de las universidades privadas de la red adventista sudamericana, caso Universidad Peruana Unión.

\section{Diseño}

El diseño de investigación es no experimental, se basó en el estudio de procesos, situaciones o hechos que ya existen en la realidad, no se manipulan ninguna variable 
y tampoco agrupan a los sujetos de la muestra siguiendo un criterio determinado. Simplemente trata de establecer el grado de mejoramiento de un sistema de referencia. Para tal efecto se implementó un modelo sistémico de mejora continua para optimizar los aspectos operativos y de modelamiento de los procesos académicos, caso: Universidad Peruana Unión. Se aplicó una adecuación del diseño de preprueba y posprueba con un solo grupo. Se llevó un seguimiento del plan de mejora, validado por la opinión de los usuarios: estudiantes y docentes (Hernández et al., 2010, p. 136).

$$
\text { G: } \quad O_{1} \quad X \quad O_{2}
$$

G: Muestra representativa de la población de estudiantes/docentes.

$\mathrm{O}_{1}$ y $\mathrm{O}_{2}$ : Son las observaciones de la valoración de la optimización de los procesos académicos que resultan respectivamente al aplicar el cuestionario al inicio y al término de la fase de mejoramiento.

$X$ : M odelo sistémico de mejora continua.

\section{Descripción del modelo sistémico de mejora continua}

La investigación se llevó a cabo con la metodología de sistemas blandos (MSB) para un modelo sistémico de mejora continua que redefine los procesos académicos a partir de las situaciones problemáticas encontradas en el funcionamiento de los procesos, a través de las siguientes fases:

\section{Situación no estructurada - planificar}

Es cuando empezamos cualquier cosa nueva que no se conoce, es el punto de inicio cuando hacemos alguna intervención sistémica, empezar a definir el sistema de referencia, los clientes, actores y dueños, cuál es su límite, analizar sus elementos. Captar la información desde encuestas hasta técnicas analíticas.

\section{Situación estructurada - planificar}

Se debe percibirla en términos fenomenológicos, hermenéuticos, epistemológicos y sistémicos.

Es aquí donde se define y grafica el sistema de referencia. También se realiza un cuadro pictográfico, bajo los términos ya mencionados, es graficar a la organización en estudio.

\section{Definiciones básicas - planificar}

Es una afirmaciónde cliente, actores, transformación, visión y entorno de la intencionalidad que le atribuimos a las reales intenciones de lo que una persona o grupo hace de la realidad.

Cuando hablamos de definiciones hablamos de sistemas relevantes, cada sistema relevante constituye una definición básica.

Es un párrafo en el cual está un verbo calificativo que plantea el proceso de transformación principal que hemos planteado.

\section{La elaboración de los modelos conceptuales - planificar}

Cada definición básica genera un modelo conceptual, que no es sino la expresión, el lenguaje sistémicoagrupación de verbos calificados y unidos gráficamenteque nos indica la manera cómo se podría llevar a cabo el proceso de transformar la realidad social.

\section{Comparación de $\mathbf{2}$ versus $\mathbf{4}$ - hacer}

Puesto que los modelos conceptuales son consecuencia de las definiciones básicas y elaboraciones mentales de procesos de transformación que pueden existir o no en la realidad, se requiere de un proceso o no de contrastación entre los modelos mentales y la realidad social que describen.

\section{C ambios factibles y deseables - verificar}

En esta etapa implica detectar qué cambio es posible llevar a cabo en la realidad.

Deben ser culturalmente factibles y sistémicamente deseables.

\section{Implantación de los cambios en el mundo real - actuar}

Es la ejecución y puesta en marcha de los cambios detectados en la etapa anterior.

\section{R esultados}

\section{Sobre las características de la muestra de estudio: estudiantes}

L a distribución de los estudiantes de la muestra según el ciclo académico es: $36,8 \%$ cursan el primer ciclo, $14.7 \%$ el tercer ciclo, $8,1 \%$ el quinto ciclo, $9,1 \%$ el séptimo ciclo y el 31.3 el décimo ciclo.

La distribución de la muestra de estudiantes por facultades es: $27 \%$ pertenecen a la Facultad de Ciencias de la Salud, 22,5\% a la Facultad de Ciencias Empresariales, el $9.1 \%$ a la Facultad de Ciencias Humanas y Educación, el $24.4 \%$ a la Facultad de Ingeniería y A rquitectura y el $16.9 \%$ a la Facultad de Teología. 
El rendimiento académico de los estudiantes de la muestra encuestada es: del $59.9 \%$ su promedio de notas es medio ( 13 a 16), del $36.2 \%$ su promedio es alto (17 a $20)$ y solamente del $3.9 \%$ su promedio de notas es bajo $(\leq 12)$.

La participación del alumno en la clase para la comprensión de los conceptos y empleo de los procedimientos es: el $60,3 \%$ casi siempre pregunta y sustenta su punto de vista, el 33,6\% siempre pregunta y sustenta su punto de vista y el $6,2 \%$ no pregunta menos discute.

Con respecto a las horas de estudio semanal empleadas por los estudiantes se tiene: el 47,2\% utilizan de 6 a 15 horas semanales, el $33,6 \%$ usan 16 o más horas semanales y el $19.2 \%$ utilizan 1 a 5 horas semanales para sus estudios.

\section{Sobre las características de la muestra de estudio: docentes}

El tiempo de servicio en la labor docente de los profesores encuestados es: el $61.5 \%$ tiene más de tres años, el $28,1 \%$ entre uno y tres años, $7.3 \%$ entre seis meses y un año y el $3.1 \%$ entre uno y seis meses.

La muestra de docentes suministradora de datos está distribuida de la siguiente manera: $21.9 \%$ pertenecen a la F acultad de Ciencias de la Salud, 18,8\% a la Facultad de Ciencias Empresariales, el $19.8 \%$ a la Facultad de Ciencias Humanas y Educación, el $27.1 \%$ a la Facultad de Ingeniería y A rquitectura y el $12.5 \%$ a la Facultad de Teología.

El $60,4 \%$ de los docentes tienen la categoría auxiliar, $24 \%$ la categoría de asociado, y el $15,6 \%$ la categoría de principal.

En cuanto al ámbito relacional en clase que brinda el docente hacia sus estudiantes: el $35,4 \%$ es facilitador, el $34,3 \%$ es abierto, el $17,7 \%$ es motivador, el $7,3 \%$ es receptivo y el $5,2 \%$ es crítico.

Con respecto al grado de compromiso con la gestión de los procesos académicos: el $51 \%$ tienen un grado de compromiso medio y el $49 \%$ un compromiso alto. $N$ ingún docente tiene un grado de compromiso bajo.

Finalmente, con respecto a la colaboración y liderazgo de los docentes: el $64,6 \%$ admite tener un liderazgo al to y el $35,4 \%$ manifiesta tener un liderazgo medio. Ningún docente encuestado valoró su liderazgo en la categoría bajo.

\section{Sobre la valoración de los procesos académicos previos y posteriores: estudiantes}

La valoración del estado de optimización del proceso académico de matrícula - aspectos estratégicos, al inicio de la aplicación del modelo sistémico de mejora continua se ubica en el nivel de desacuerdo y al término en el nivel de acuerdo. La valoración media del proceso de matrícula - aspectos estratégicos en la primera vez fue de 2,36, con una desviación típica de 0,539, y en una segunda vez, después de aplicarse el modelo sistémico de mejora continua, fue de 4,22, con una desviación típica de 0,418 . El $50 \%$ de los estudiantes encuestados al inicio valoran los aspectos estratégicos por debajo de 2 y al término por encima de 4. Al inicio la valoración de los estudiantes en este aspecto están conglomerados en las categorías completamente en desacuerdo 0 en desacuerdo o neutral con un $97.7 \%$ del total; al término están conglomerados en las categorías de acuerdo 0 completamente de acuerdo en el $100 \%$.

La valoración del estado de optimización del proceso académico de matrícula - aspectos de estructura, al inicio de la aplicación del modelo sistémico de mejora continua se ubica en el nivel de desacuerdo y al término en el nivel de acuerdo. La valoración media del proceso de matrícula - aspectos de estructura en la primera vez fue de 2,43 , con una desviación típica de 0,564 , y en una segunda vez, después de aplicarse el modelo sistémico de mejora continua, fue de 4,28, con una desviación típica de 0,451 . El $50 \%$ de los estudiantes encuestados al inicio valoran los aspectos de estructura por debajo de 2 y al término por encima de 4. Al inicio la valoración de los estudiantes en este aspecto están conglomerados en las categorías completamente en desacuerdo 0 en desacuerdo o neutral con un $98 \%$ del total; al término están conglomerados en las categorías de acuerdo o completamente de acuerdo en el $100 \%$.

La valoración del estado de optimización del proceso académico de matrícula - aspectos de procesos intermedios, al inicio de la aplicación del modelo sistémico de mejora continua se ubica en el nivel de desacuerdo y al término en el nivel de acuerdo. La valoración media del proceso de matrícula - aspectos de procesos intermedios en la primera vez fue de 2,38, con una desviación típica de 0,585, y en una segunda vez, después de aplicarse el modelo sistémico de mejora continua, fue de 4,15, con una desviación típica de 0,361. El $50 \%$ de los estudiantes encuestados al inicio valoran los aspectos de procesos intermedios por debajo de 2 y al término por encima de 4. Al inicio la valoración de los estudiantes en este aspecto están conglomerados en las categorías completamente en desacuerdo 0 en desacuerdo o neutral con un $98 \%$ del total; al término están conglomerados en las categorías de acuerdo o completamente de acuerdo en el $100 \%$. 
La valoración del estado de optimización del proceso académico de matrícula - aspectos de resultado, al inicio de la aplicación del modelo sistémico de mejora continua se ubica cerca del nivel neutral y al término en el nivel de acuerdo. La valoración media del proceso de matrícula - aspectos de resultado en la primera vez fue de 2,66 , con una desviación típica de 0,648 , y en una segunda vez, después de aplicarse el modelo sistémico de mejora continua, fue de 4,25 , con una desviación típica de 0,454 . El $50 \%$ de los estudiantes encuestados al inicio valoran los aspectos de resultado por debajo de 3 y al término por encima de 4. Al inicio la valoración de los estudiantes en este aspecto están conglomerados en las categorías en desacuerdo o neutral con un $91.9 \%$ del total; al término están conglomerados en las categorías de acuerdo o completamente de acuerdo en un $99 \%$ del total.

La valoración del estado de optimización del proceso académico de evaluación docente - aspectos estratégicos, al inicio de la aplicación del modelo sistémico de mejora continua se ubica en un nivel cerca a neutral y al término en el nivel de acuerdo. la valoración media del proceso de evaluación docente - aspectos estratégicos en la primera vez fue de 2,36, con una desviación típica de 0,539 , y en una segunda vez, después de aplicarse el modelo sistémico de mejora continua, fue de 4,05 , con una desviación típica de 0,304. El 50\% de los estudiantes encuestados al inicio valoran los aspectos estratégicos por debajo de 3 y al término por encima de 4 . Al inicio la valoración de los estudiantes en este aspecto están conglomerados en las categorías completamente en desacuerdo 0 en desacuerdo o neutral con un $95.4 \%$ del total; al término están conglomerados en las categorías de acuerdo o completamente de acuerdo con un $97,8 \%$ del total.

La valoración del estado de optimización del proceso académico de evaluación docente - aspectos de estructura, al inicio de la aplicación del modelo sistémico de mejora continua se ubica cerca del nivel de desacuerdo y al término en el nivel de acuerdo. La valoración media del proceso de evaluación docente aspectos de estructura en la primera vez fue de 2.50 , con una desviación típica de 0,602 , y en una segunda vez, después de aplicarse el modelo sistémico de mejora continua, fue de 3,98, con una desviación típica de 0,347. El $50 \%$ de los estudiantes encuestados al inicio valoran los aspectos de estructura por debajo de 2 y al término por encima de 4. Al inicio la valoración de los estudiantes en este aspecto están conglomerados en las categorías en desacuerdo o neutral con un $96,1 \%$ del total; al término están conglomerados en las categorías de acuerdo o completamente de acuerdo con un $92.8 \%$ del total.

La valoración del estado de optimización del proceso académico de evaluación docente - aspectos de procesos intermedios, al inicio de la aplicación del modelo sistémico de mejora continua se ubica cerca del nivel de desacuerdo y al término en el nivel de acuerdo. La valoración media del proceso de evaluación docente aspectos de procesos intermedios en la primera vez fue de 2,51 , con una desviación típica de 0,618 , y en una segunda vez, después de aplicarse el modelo sistémico de mejora continua, fue de 4, con una desviación típica de 0,338 . El $50 \%$ de los estudiantes encuestados al inicio valoran los aspectos de procesos intermedios por debajo de 3 y al término por encima de 4 . Al inicio la valoración de los estudiantes en este aspecto están conglomerados en las categorías en desacuerdo o neutral con un $96.1 \%$ del total; al término están conglomerados en las categorías de acuerdo o completamente de acuerdo con un $94.1 \%$ del total.

La valoración del estado de optimización del proceso académico de evaluación docente - aspectos de resultado, al inicio de la aplicación del modelo sistémico de mejora continua se ubica cerca del nivel de desacuerdo y al término en el nivel de acuerdo. La valoración media del proceso de evaluación docente - aspectos de resultado en la primera vez fue de 2,57 , con una desviación típica de 0,630 , y en una segunda vez, después de aplicarse el modelo sistémico de mejora continua, fue de 4,11, con una desviación típica de 0,432. El 50\% de los estudiantes encuestados al inicio valoran los aspectos de resultado por debajo de 3 y al término por encima de 4 . Al inicio la valoración de los estudiantes en este aspecto están conglomerados en las categorías completamente en desacuerdo 0 en desacuerdo o neutral con un $93.8 \%$ del total; al término están conglomerados en las categorías de acuerdo o completamente de acuerdo con un $95.7 \%$ del total.

La valoración del estado de optimización del proceso académico de graduación - aspectos estratégicos, al inicio de la aplicación del modelo sistémico de mejora continua se ubica en el nivel de desacuerdo y al término en el nivel de acuerdo. La valoración media del proceso de graduación - aspectos estratégicos en la primera vez fue de 2,50, con una desviación típica de 0,574, y en una segunda vez, después de aplicarse el modelo sistémico de mejora continua, fue de 4,13 , con una desviación típica de 0,394 . El $50 \%$ de los estudiantes encuestados al inicio valoran los aspectos estratégicos por debajo de 2 y 
al término por encima de 4. Al inicio la valoración de los estudiantes en este aspecto están conglomerados en las categorías completamente en desacuerdo 0 en desacuerdo o neutral con un $96.4 \%$ del total; al término están conglomerados en las categorías de acuerdo 0 completamente de acuerdo con un $98 \%$ del total.

La valoración del estado de optimización del proceso académico de graduación - aspectos de estructura, al inicio de la aplicación del modelo sistémico de mejora continua se ubica en el nivel de desacuerdo y al término en el nivel de acuerdo. La valoración media del proceso de graduación - aspectos de estructura en la primera vez fue de 2,40 , con una desviación típica de 0,583, y en una segunda vez, después de aplicarse el modelo sistémico de mejora continua, fue de 4,13 , con una desviación típica de 0,418 . El $50 \%$ de los estudiantes encuestados al inicio valoran los aspectos de estructura por debajo de 2 y al término por encima de 4. Al inicio la valoración de los estudiantes en este aspecto están conglomerados en las categorías completamente en desacuerdo 0 en desacuerdo o neutral con un $97.1 \%$ del total; al término están conglomerados en las categorías de acuerdo 0 completamente de acuerdo con un $97.1 \%$ del total.

La valoración del estado de optimización del proceso académico de graduación - aspectos de procesos intermedios, al inicio de la aplicación del modelo sistémico de mejora continua se ubica en el nivel de desacuerdo y al término en el nivel de acuerdo. la valoración media del proceso de graduación - aspectos de procesos intermedios en la primera vez fue de 2,36, con una desviación típica de 0,574 , y en una segunda vez, después de aplicarse el modelo sistémico de mejora continua, fue de 4,16, con una desviación típica de 0,480. El $50 \%$ de los estudiantes encuestados al inicio valoran los aspectos de procesos intermedios por debajo de 2 y al término por encima de 4 . A I inicio la valoración de los estudiantes en este aspecto están conglomerados en las categorías completamente en desacuerdo 0 en desacuerdo o neutral con un $97,1 \%$ del total; al término están conglomerados en las categorías de acuerdo 0 completamente de acuerdo con un $95.1 \%$ del total.

La valoración del estado de optimización del proceso académico de graduación - aspectos de resultado, al inicio de la aplicación del modelo sistémico de mejora continua se ubica el nivel de desacuerdo y al término en el nivel de acuerdo. La valoración media del proceso de graduación - aspectos de resultado en la primera vez fue de 2,37, con una desviación típica de 0,599, y en una segunda vez, después de aplicarse el modelo sistémico de mejora continua, fue de 4,26 , con una desviación típica de 0,504 . El $50 \%$ de los estudiantes encuestados al inicio valoran los aspectos de resultado por debajo de 2 y al término por encima de 4 . Al inicio la valoración de los estudiantes en este aspecto están conglomerados en las categorías en desacuerdo o neutral con un $95.1 \%$ del total; al término están conglomerados en las categorías de acuerdo o completamente de acuerdo en un $97,1 \%$ del total.

La valoración del estado de optimización del proceso académico de evaluación discente - aspectos estratégicos, al inicio de la aplicación del modelo sistémico de mejora continua se ubica en el nivel de desacuerdo y al término en el nivel de acuerdo. La valoración media del proceso de evaluación discente aspectos estratégicos en la primera vez fue de 2,49, con una desviación típica de 0,591, y en una segunda vez, después de aplicarse el modelo sistémico de mejora continua, fue de 4,05, con una desviación típica de 0,418. El $50 \%$ de los estudiantes encuestados al inicio valoran los aspectos estratégicos por debajo de 2 y al término por encima de 4. Al inicio la valoración de los estudiantes en este aspecto están conglomerados en las categorías completamente en desacuerdo 0 en desacuerdo o neutral con un $96.4 \%$ del total; al término están conglomerados en las categorías de acuerdo o completamente de acuerdo con un $93,5 \%$ del total.

La valoración del estado de optimización del proceso académico de evaluación discente - aspectos de estructura, al inicio de la aplicación del modelo sistémico de mejora continua se ubica cerca del nivel neutro y al término en el nivel de acuerdo. La valoración media del proceso de evaluación discente - aspectos de estructura en la primera vez fue de 2.67, con una desviación típica de 0,636 , y en una segunda vez, después de aplicarse el modelo sistémico de mejora continua, fue de 4,16 , con una desviación típica de 0,393. El 50\% de los estudiantes encuestados al inicio valoran los aspectos de estructura por debajo de 3 y al término por encima de 4 . Al inicio la valoración de los estudiantes en este aspecto están conglomerados en las categorías en desacuerdo o neutral con un $92,2 \%$ del total; al término están conglomerados en las categorías de acuerdo o completamente de acuerdo con un $99 \%$ del total.

La valoración del estado de optimización del proceso académico de evaluación discente - aspectos de procesos intermedios, al inicio de la aplicación del modelo sistémico de mejora continua se ubica en el nivel de desacuerdo y al término en el nivel de acuerdo. La 
valoración media del proceso de evaluación discente aspectos de procesos intermedios en la primera vez fue de 2,39, con una desviación típica de 0,614 , y en una segunda vez, después de aplicarse el modelo sistémico de mejora continua, fue de 4,01, con una desviación típica de 0,348 . El $50 \%$ de los estudiantes encuestados al inicio valoran los aspectos de procesos intermedios por debajo de 2 y al término por encima de 4 . Al inicio la valoración de los estudiantes en este aspecto están conglomerados en las categorías completamente en desacuerdo 0 en desacuerdo o neutral con un $95.4 \%$ del total; al término están conglomerados en las categorías de acuerdo o completamente de acuerdo con un $94.4 \%$ del total.

La valoración del estado de optimización del proceso académico de evaluación discente - aspectos de resultado, al inicio de la aplicación del modelo sistémico de mejora continua se ubica cerca del nivel neutral y al término en el nivel de acuerdo. La valoración media del proceso de evaluación discente - aspectos de resultado en la primera vez fue de 2,56, con una desviación típica de 0,665 , y en una segunda vez, después de aplicarse el modelo sistémico de mejora continua, fue de 4,16 , con una desviación típica de 0,457. El 50\% de los estudiantes encuestados al inicio valoran los aspectos de resultado por debajo de 3 y al término por encima de 4 . Al inicio la valoración de los estudiantes en este aspecto están conglomerados en las categorías completamente en desacuerdo 0 en desacuerdo o neutral con un $92.8 \%$ del total; al término están conglomerados en las categorías de acuerdo o completamente de acuerdo con un $96.4 \%$ del total.

\section{Sobre la valoración de los procesos académicos previos y posteriores: docentes}

La valoración del estado de optimización del proceso académico de evaluación docente - aspectos estratégicos, al inicio de la aplicación del modelo sistémico de mejora continua se ubica en el nivel neutral y al término en el nivel de acuerdo. La valoración media del proceso de evaluación docente - aspectos estratégicos en la primera vez fue de 2,80, con una desviación típica de 0,592 , y en una segunda vez, después de aplicarse el modelo sistémico de mejora continua, fue de 4,13, con una desviación típica de 0,332. El 50\% de los docentes encuestados al inicio valoran los aspectos estratégicos por debajo de $3 \mathrm{y}$ al término por encima de 4 . Al inicio la valoración de los docentes en este aspecto están conglomerados en las categorías completamente en desacuerdo o en desacuerdo o neutral con un $91.7 \%$ del total; al término están conglomerados en las categorías de acuerdo o completamente de acuerdo en el $100 \%$.

La valoración del estado de optimización del proceso académico de evaluación docente - aspectos de estructura, al inicio de la aplicación del modelo sistémico de mejora continua se ubica en el nivel neutral y al término en el nivel de acuerdo. La valoración media del proceso de evaluación docente - aspectos de estructura en la primera vez fue de 2,76 , con una desviación típica de 0,538 , y en una segunda vez, después de aplicarse el modelo sistémico de mejora continua, fue de 4,21 , con una desviación típica de 0,408 . El $50 \%$ de los docentes encuestados al inicio valoran los aspectos de estructura por debajo de 3 y al término por encima de 4 . Al inicio la valoración de los docentes en este aspecto están conglomerados en las categorías completamente en desacuerdo 0 en desacuerdo o neutral con un $95.8 \%$ del total; al término están conglomerados en las categorías de acuerdo o completamente de acuerdo en el $100 \%$.

La valoración del estado de optimización del proceso académico de evaluación docente - aspectos de procesos intermedios, al inicio de la aplicación del modelo sistémico de mejora continua se ubica en el nivel de desacuerdo y al término en el nivel de acuerdo. La valoración media del proceso de evaluación docente aspectos de procesos intermedios en la primera vez fue de 2,41 , con una desviación típica de 0,674 , y en una segunda vez, después de aplicarse el modelo sistémico de mejora continua, fue de 4,13 , con una desviación típica de 0,332. El 50\% de los docentes encuestados al inicio valoran los aspectos de procesos intermedios por debajo de 2 y al término por encima de 4 . Al inicio la valoración de los docentes en este aspecto están conglomerados en las categorías completamente en desacuerdo 0 en desacuerdo o neutral con un $97.9 \%$ del total; al término están conglomerados en las categorías de acuerdo o completamente de acuerdo en el $100 \%$.

La valoración del estado de optimización del proceso académico de evaluación docente - aspectos de resultado, al inicio de la aplicación del modelo sistémico de mejora continua se ubica cerca del nivel neutral y al término en el nivel de acuerdo. La valoración media del proceso de evaluación docente - aspectos de resultado en la primera vez fue de 2,94, con una desviación típica de 0,477 , y en una segunda vez, después de aplicarse el modelo sistémico de mejora continua, fue de 4,38, con una desviación típica de 0,487. El $50 \%$ de los docentes encuestados al inicio valoran los aspectos de resultado por debajo de $3 \mathrm{y}$ al término por encima de 4 . Al inicio 
la valoración de los docentes en este aspecto están conglomerados en las categorías en desacuerdo o neutral con un $91.7 \%$ del total; al término están conglomerados en las categorías de acuerdo o completamente de acuerdo en el $100 \%$.

La valoración del estado de optimización del proceso académico de evaluación discente - aspectos estratégicos, al inicio de la aplicación del modelo sistémico de mejora continua se ubica en el nivel neutral y al término en el nivel de acuerdo. La valoración media del proceso de evaluación discente - aspectos estratégicos en la primera vez fue de 2,36, con una desviación típica de 0,539, y en una segunda vez, después de aplicarse el modelo sistémico de mejora continua, fue de 4,26, con una desviación típica de 0,441. El $50 \%$ de los docentes encuestados al inicio valoran los aspectos estratégicos por debajo de 3 y al término por encima de 4. Al inicio la valoración de los docentes en este aspecto están conglomerados en las categorías completamente en desacuerdo 0 en desacuerdo 0 neutral con un $93.8 \%$ del total; al término están conglomerados en las categorías de acuerdo o completamente de acuerdo en el $100 \%$.

La valoración del estado de optimización del proceso académico de evaluación discente - aspectos de estructura, al inicio de la aplicación del modelo sistémico de mejora continua se ubica en el nivel neutral y al término en el nivel de acuerdo. La valoración media del proceso de evaluación discente - aspectos de estructura en la primera vez fue de 3.04, con una desviación típica de 0,710 , y en una segunda vez, después de aplicarse el modelo sistémico de mejora continua, fue de 4,51, con una desviación típica de 0,503. El 50\% de los docentes encuestados al inicio valoran los aspectos de estructura por debajo de $3 \mathrm{y}$ al término igual a 5 . Al inicio la valoración de los docentes en este aspecto están conglomerados en las categorías en desacuerdo o neutral con un $72.9 \%$ del total; al término están conglomerados en las categorías de acuerdo o completamente de acuerdo en el $100 \%$.

La valoración del estado de optimización del proceso académico de evaluación discente - aspectos de procesos intermedios, al inicio de la aplicación del modelo sistémico de mejora continua se ubica en el nivel de desacuerdo y al término en el nivel de acuerdo. La valoración media del proceso de evaluación discente aspectos de procesos intermedios en la primera vez fue de 2,5, con una desviación típica de 0,543 , y en una segunda vez, después de aplicarse el modelo sistémico de mejora continua, fue de 4,13 , con una desviación típica de 0,332. El 50\% de los docentes encuestados al inicio valoran los aspectos de procesos intermedios por debajo de 2 y al término por encima de 4 . Al inicio la valoración de los docentes en este aspecto están conglomerados en las categorías en desacuerdo o neutral con un $97.9 \%$ del total; al término están conglomerados en las categorías de acuerdo o completamente de acuerdo en el $100 \%$.

La valoración del estado de optimización del proceso académico de evaluación discente - aspectos de resultado, al inicio de la aplicación del modelo sistémico de mejora continua se ubica en el nivel neutral y al término en el nivel de acuerdo. la valoración media del proceso de evaluación discente - aspectos de resultado en la primera vez fue de 3,10 , con una desviación típica de 0,688 , y en una segunda vez, después de aplicarse el modelo sistémico de mejora continua, fue de 4,42 , con una desviación típica de 0,496. El 50\% de los docentes encuestados al inicio valoran los aspectos de resultado por debajo de 3 y al término por encima de 4 . Al inicio la valoración de los docentes en este aspecto están conglomerados en las categorías completamente en desacuerdo 0 en desacuerdo o neutral con un $95.8 \%$ del total; al término están conglomerados en las categorías de acuerdo o completamente de acuerdo en el $100 \%$.

\section{Conclusión}

\section{Sobre la incidencia del modelo sistémico de mejora continua: estudiantes}

Utilizamos la prueba relativa a la diferencia de dos medias, dada por el estadístico t de Student, para la prueba de las cuatro hipótesis específicas y la general, con un nivel de significación de $\alpha=0,01$. Los subíndices 1 y 2 de la media $\mu$, respectivamente designan el promedio de las observaciones del inicio y del término de la aplicación sistémica de mejora continua de los procesos académicos de la UPeU. La formulación de cada hipótesis estadística es: $H_{0}: \mu_{2} \leq \mu_{1}, H_{1}: \mu_{2}>\mu_{1}$

- Al término de la aplicación del modelo sistémico de mejora continua, con una $t$ de Student de 83,635 y con 306 g.l., la optimización del proceso de matrícula aumenta con una significación del 5\%. Luego, el modelo sistémico de mejora continua es eficiente en la optimización del proceso de matrícula en universidades privadas de la red universitaria adventista sudamericana: caso Universidad Peruana Unión. 
- Al término de la aplicación del modelo sistémico de mejora continua, con una $t$ de Student de 93,236 y con 306 g.l., la optimización del proceso de evaluación docente aumenta con una significación del 5\%. Luego, el modelo sistémico de mejora continua es eficiente en la optimización del proceso de evaluación docente en universidades privadas de la red universitaria adventista sudamericana: caso Universidad Peruana Unión.

- Al término de la aplicación del modelo sistémico de mejora continua, con una $t$ de Student de 137,121 y con 306 g.l., la optimización del proceso de graduación aumenta con una significación del 5\%. Luego, el modelo sistémico de mejora continua es eficiente en la optimización del proceso de graduación en universidades privadas de la red universitaria adventista sudamericana: caso Universidad Peruana Unión.

- Al término de la aplicación del modelo sistémico de mejora continua, con una $t$ de Student de 88,918 y con 306 g.l., la optimización del proceso de evaluación discente aumenta con una significación del 5\%. Luego, el modelo sistémico de mejora continua es eficiente en la optimización del proceso de evaluación discente en universidades privadas de la red universitaria adventista sudamericana: caso Universidad Peruana Unión.

- Al término de la aplicación del modelo sistémico de mejora continua, con una $t$ de Student de 125,331 y con 306 g.l., la optimización de los procesos académicos aumenta con una significación del 5\%. Luego, el modelo sistémico de mejora continua es eficiente en la optimización de los procesos académicos en universidades privadas de la red universitaria adventista sudamericana: caso Universidad Peruana U nión.

\section{Sobre la incidencia del modelo sistémico de mejora continua: docentes}

Utilizamos la prueba relativa a la diferencia de dos medias, dada por el estadístico t de Student, para la prueba de las cuatro hipótesis específicas y la general, con un nivel de significación de $\alpha=0,01$. Los subíndices 1 y 2 de la media $\mu$, respectivamente designan el promedio de las observaciones del inicio y del término de la aplicación sistémica de mejora continua de los procesos académicos de la UPeU. La formulación de cada hipótesis estadística es: $H_{0}: \mu_{2} \leq \mu_{1}, H_{1}: \mu_{2}>\mu_{1}$

- Al término de la aplicación del modelo sistémico de mejora continua, con una $t$ de Student de 22,192 y con 95 g.l., la optimización del proceso de evaluación docente aumenta con una significación del $5 \%$. Luego, el modelo sistémico de mejora continua es eficiente en la optimización del proceso de evaluación docente en universidades privadas de la red universitaria adventista sudamericana: caso Universidad Peruana Unión.

- Al término de la aplicación del modelo sistémico de mejora continua, con una t de Student de 22,951 y con 95 g.l., la optimización del proceso de evaluación discente aumenta con una significación del $5 \%$. Luego, el modelo sistémico de mejora continua es eficiente en la optimización del proceso de evaluación discente en universidades privadas de la red universitaria adventista sudamericana: caso Universidad Peruana Unión.

- Al término de la aplicación del modelo sistémico de mejora continua, con una t de Student de 23,958 y con 95 g.l., la optimización de los procesos académicos aumenta con una significación del $5 \%$. Luego, el modelo sistémico de mejora continua es eficiente en la optimización de los procesos académicos en universidades privadas de la red universitaria adventista sudamericana: caso Universidad Peruana Unión. 


\section{Referendias}

Ancinas, J. (2006). Administración total del mejoramiento continuo. La nueva generación. Colombia: M cG raw Hill Interamericana.

Álvarez, F. (1987). Administración, una introducción al estudio de la Administración. Córdoba: Sociedad para Estudios Pedagógicos A rgentinos.

A rcudia, C. (2006). La empresa y sus operaciones bajo un enfoque de sistemas. M éxico: A cadémica.

A rnold, M. (1989). Teoría de Sistemas, Nuevos Paradigmas: Enfoque de Niklas Luhmann. Paraguay: Sociedad Paraguaya de Sociología.

A shby, W. (1984). Sistemas y sus Medidas de Información. España: Alianza.

Bastonsi, M. (2004). La toma de decisiones en la organización. E spaña: A riel.

Beer, S. (1966). "Decision and control the meaning of operational research and management cybernetics". K nowledge and Process $M$ anagement, vol. 42, no.2, pp. 31-36

Bertalanffy, L. (1991). Teoría General de Sistemas. B uenos A ires: Fondo de Cultura Económica.

Biazzi, M. (2007). Instituciones públicas de enseñanza superior: mejora de procesos administrativos en instituciones públicas. Brasil: McGraw Hill Interamericana.

Blanchard, K. (1991). Administración de Ingeniería de Sistemas. M éxico: Limusa.

Boulding, E. (1954). The Organizational Revolution: A Study in the Ethics of Economic Organization. Estados U nidos: Prentice Hall.

Calvo, S. (2006). F actores determinantes de la calidad percibida. España: Universidad Complutense de M adrid.

Castro, D. (2008, January 23). Los órganos unipersonales territoriales de gestión académica en la universidad: contexto, problemáticas y propuestas de mejora. U niversitat A utónoma de B arcel ona. Retrieved from

http://www.tesisenred.net/handle/10803/5050

Coelho,F, Romero,M , \& Y áber,G . (2005). Indicadores de desempeño clave para programas académicos de posgrado. Investigación y Posgrado, 20(2), 123153. Retrieved from http://www.scielo.org.ve/scielo.php?script=sci_artt ext\& pid $=S 1316$ $00872005000200005 \&$ Ing $=e s \&$ nrm=iso \& tlng=es

Chavaleta, N. (1994) La administración del personal académico, su formación y superación. Revista Cubana de Educación Superior (La Habana) 14, (1):61-70.

Checkland, P. y Scholes, J. (1995). Soft Systems Methodology in Action. England: Wiley.

Checkland, P. (1992). Pensamiento de Sistemas, Práctica de Sistemas. M éxico: Limusa.

Clark, C. (2002). Investigación en Psicología. M éxico: Oxford University Press.

Davenport, T. H., y Beers, M. C., (1995). "Managing information about processes". J ournal of Management Information Systems, vol. 12 , no. 1, pp. 57-80.

Davenport, T. H., y Short, J. E., (1990). "The new industrial engineering: Information Technology and Business Process Redesign". Sloan M anagement Review, vol. 31, no. 4, pp. 11-27

Davenport, T. Jarvenpaa, S. L., Beers, M., (1996). "Improving Knowledge Work Processes". Sloan $M$ anagement Review, vol. 37, no. 4, pp. 53-65.

Deming, W. (1989). Calidad, productividad y competitividad: la salida de la crisis. Madrid: Ediciones Díaz de Santos.

Ehrenberg, R., y Stupak, R., (1994). "Total Quality $M$ anagement: Its relationship to administrative theory and organizational behaviour in the public sector". Public Administration Quartely, pp. 75-98. 
Fayol, H. (1916). Administración Industrial y General. Buenos Aires, Argentina, Editorial El A teneo, Reimpresión en 1982.

Fernández, A . (2001). Organización de los contenidos de sistemas de información. Cuba: A cimed.

Fiorelly, J., y Feller, R., (1994). "Re-engineering TQM and Work redesign: An integrate approach to continuous organizational excellence". Public Administration Quartely, pp. 54-63.

Gallaga, R. (2005). Teoría de las relaciones humanas. Perú: Ilustrados.

Garzon C. (2012). La mejora continua y la calidad en instituciones de formación profesional. El proceso de enseñanza-aprendizaje. U niversitat Autónoma de Barcelona. Retrieved from http://www.tesisenred.net/handle/10803/96828

Goldratt, E. (2005). La Meta. 2o Edición, M éxico, Ediciones Regiomontanas.

Guerrero, F. (2005). El uso de manuales de procedimientos para contribuir a la optimización. Perú: Ilustrados.

Hammer, M. (1990). "Reengineering work: Don't automate, obliterate". Harvard Business Review, no. 90406, pp. 104-112.

Hayes, E. (2002). Cómo medir la satisfacción del cliente. España: Limpergraf.

Heizer, J., y Render, B. (2001). O perations M anagement. $\mathrm{N}$ ew Jersey, Prentice $\mathrm{Hall}$.

Checkland, P. (1981). Systems Thinking Systems Practice. Chichester, J ohn Wiley.

Hernández, R., Fernández, C. y Baptista, L. (2010). M etodología de la investigación. M éxico: M cGraw - Hill.

Kast, F., y Rosenzweig, J. E., (1972). "General Systems Theory applications for Organization and Management". A cademy of M anagement Review, vol.15, no. 4, pp. 447-465.

Kettinger, W., Teng, T., Guha, S., (1997). "Business Process Change: A study of Methodologies,
Techniques, and tools". MIS-Quarterly, vol. 21, no.1, pp. 55-80

Lee, R., y Dale, B., (1998). "Business Process $M$ anagement: A review and evaluation". Business Process $M$ anagement J ournal, vol. 4, no. 3, pp. 214223.

Lefcovich, M. (2005). Gestión de calidad para la excelencia. Perú: Ilustrados.

López, M . (2008). Sistemas de soporte de decisiones y su implementación tecnológica: gestión por categorías. España: Universidad Complutense de Madrid.

Lind, A.; M archal, G. y Mason, D. (2004). Estadística para administración y economía. Colombia: Alfaomega Grupo Editor, S.A. de C.V .

Lin, C., Frank Chen, F., W an, H., M in Chen, $Y$., \& K uriger, G. (2013). Continuous improvement of knowledge management systems using Six Sigma methodology. Robotics and Computer-Integrated M anufacturing, 29(3), 95-103. doi:10.1016/j.rcim.2012.04.018

Machado, L. (1996). El objeto relación Universidad sociedad como sistema, Revista cubana de educación superior (La H abana) 1(3), 40-52.

Martínez, B. (2006). La formación de la empresa, ingeniería de los procesos educativos. España: Universidad Politécnica de V alencia.

Miralles, L. (2004). ¿Está su empresa realmente orientada al cliente? España: D eusto.

M orera, J. (2006). Estadística para la toma de decisiones en el servicio al cliente. Perú: Ilustrados.

Ojalvo, V. 1994. Determinantes de la relación educativa. CEPES. Universidad de La Habana.

Oliveira, A. (2006). Cambios en la organización del trabajo docente: consecuencias para los profesores. M éxico: Educativa.

Ostroff, F. (1999). The horizontal organization. New Y ork. N.Y ., Oxford U niversity Press. 
Pablos, C. (2006). Determinantes organizativos de la capacidad de adopción de tecnologías de información en empresas del sector asegurador español. España: Universidad Complutense de $M$ adrid.

Parada, 0. (2006). La evaluación del servicio logístico: una alternativa para la mejora de la eficiencia y la satisfacción del cliente. Perú: Ilustrados.

Pelayo, C. (2005). Las principales teorías administrativas y sus principales enfoques. Perú: Ilustrados.

Peralta, M. (2005). Sistemas de Información. Perú: Ilustrados.

Rodríguez, D. (1994). Teoría de sistemas y gestión de las organizaciones. Lima: Instituto Andino de Sistemas.

Rodríguez, R. (1992). La Sistémica. Los Sistemas Blandos y los Sistemas de información. Lima: Instituto A ndino de Sistemas.

Rodríguez-Ulloa, R., \& Páucar-Cáceres, A . (2005). Soft System Dynamics Methodology (SSDM): Combining Soft Systems M ethodology

(SSM) and System Dynamics (SD). Systemic Practice and Action Research, 18(3), 303-334 LA English. doi:10.1007/s11213-005-4816-7

Rojas, H. (2005). Mejoramiento de la calidad de procesos mediante la orientación al cliente y el compromiso de la empresa. Perú: Ilustrados.

Sánchez, J. (1992). La aproximación contingente en la teoría organizacional: hacia un nuevo enfoque. España: Organizaciones.

Senge, P. (1990). La quinta disciplina. N ew Y ork. N.Y., Double Day.

Suárez, T. (2006). La organización académica de las universidades públicas: procesos académicos. $M$ éxico: A cadémica.

Suárez, F. (2008, May 22). La Sostenibilidad de la M ejora Continua de Procesos en la Administración Pública. Un estudio en los Ayuntamientos españoles. U niversitat Ramon Llull. Retrieved from http://www.tesisenred.net/handle/10803/9178
Siau, K., \& Tan, X. (2005). Improving the quality of conceptual modeling using cognitive mapping techniques. Data \& Knowledge Engineering, 55(3), 343-365. Retrieved from http://www.sciencedirect.com/science? ob=Gatewa yURL\&_origin $=$ ScienceSearch $\&$ method=citation Search\&_piikey $=S 0169023 X 04002319 \&$ _version $=$ $1 \&$ returnURL $=$ http://www.scirus.com/srsapp/\& m $\mathrm{d} 5=4 \mathrm{ca} 962 \mathrm{~d} 9 \mathrm{de} 7 \mathrm{~b} 7 \mathrm{~b} 7 \mathrm{a} 84 \mathrm{fa}$ 365e46a45ae8

Taylor, F. (1911). Principios de la Administración Científica. Buenos, A ires, Argentina., Editorial El A teneo. Reimpresión 1979.

Taylor, S. y Bogdan, R., (1984). Introduction to qualitative research methods. The search for meanings. N ew $Y$ ork, NY, J ohn W iley and Sons.

Tinnila, M. (1995). "Strategic perspective to business process redesign". M anagement Decision, vol. 33, no. 3, pp. 25-34

Trist, E. (1989). "The assumption of Ordinariness as a Denial Mechanism: Innovation and Conflict in a Coal M ine". Human Resource M anagement, vol. 28, no. 2.

Vega, P. (2006). Teoría de sistemas y evaluación de programas sociales. Chile: Red Última Década.

Walton, M. (2004). El método Deming en la práctica. B ogotá: Editorial Norma.

Weimei, Z., \& Feng-e, T. (2012). A nalysis of Performance $M$ anagement in Small and Medium Enterprises. IERI Procedia, 1, 8-12. doi:10.1016/j.ieri.2012.06.003

Wilson, B. (1984). Systems Concepts, Methodologies and A plications. Estados U nidos: W iley.

Zairi, M. (1997). "Business Process Management: A boundaryless approach to modern competitiveness". Business Process Management J ournal, vol. 3, no. 1, pp. 64-80. 